\title{
TABLE DES MATIÈRES DU TOME DEUX
}

\author{
LIVRE DEUX
}

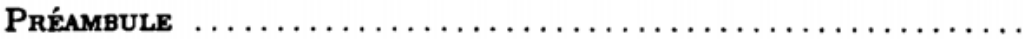

Chapitre PREMIER. - L'action ÉCONOMtQue de L'ARMÉE RÉvOlUTIONNAIRE PARISIENNE $\ldots \ldots \ldots \ldots \ldots \ldots \ldots \ldots \ldots \ldots \ldots$

I. - La géographie du ravitaillement de Paris, p. 370. - II. - Le désordre administratif et la compétence des autorités dans les missions économiques, p. 388. - III. - Les conditions saisonnières des missions économiques, p. 391. - IV. - I.es révolutionnaires et le ravitaillement de Paris, p. 394. - V. - Les révolutionnaires vus par les paysans, p. 398. - VI. - La police du ravitaillement, p. 405: Exécution des réquisitions et protections des arrivages, p. 406; (2) Mobilisation économique et police rurale, p. 416; (3) Activités individuelles, p. 419.

Chapitre II. - Défense nationale et ravttaillement urbann: L'ACTION ÉCONOMTQUE DES FORCES DÉPARTEMENTALES ET COMMUNALES

I. - Problèmes de circulation, p. 428. - II. - La crainte de manquer et les entraves humaines, p. 432. -- III. - Le ravitaillement des armées, p. 445. - IV. - Les forces révolutionnaires au service du ravitaillement communal, p. 451. - V. - Réalisations économiques des forces révolutionnaires, p. 456.

Chapitre III. - L'Armée parisienne et la répression $\ldots \ldots \ldots$

I. - Consignes officielles et attitudes personnelles, p. 468 . II. - Les arrestations, p. 474; (1) L'aire géographique et le bilan approximatif des arrestations, p. 476; (2) Motifs des arrestations et catégories politiques et sociales des personnes arrêtées, p. 481; (3) Les responsabilités administratives des arrestations : arrestations arbitraires, p. 491. - III. - Exemples des missions répressives, p. 504 ; (1) L'armée révolutionnaire et l'affaire de Vermenton, p. 507; (2) Les affaires de Villeblevin et l'expédition de ventôse an II, p. 514 ; (3) Les expéditions de Jagny et les incohérences de la politique répressive du gouvernement, p. 528; (4) L'arrestation de 
Thunot et de Boisgirault à Compiègne, p. 532. - IV. - La Grande Répression : le dossier lyonnais, p. 536. - V. - La justice révolutionnaire, p. 541.

Chapttre IV. - L'action RÉpressive des armées départementales

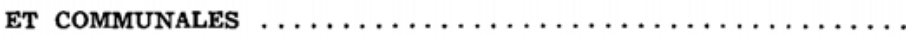

I. - Anarchie et répression, p. 547. - II. - Consignes de sévérité et volonté punitive, p. 554. - III. - Les arrestations, p. 560. IV. - Les Grands Moyens : les noyades de Nantes, les fusillades du Morbihan, l'invasion de l'Aveyron par l'armée de Viton, p. 576. V. - Les activités individuelles, p. 592.

Chapitre V. - L'ACTION POLITIQUE DES ARMÉEs RÉVOLUTIONNAIRES.

I. - L'armée parisienne dans la politique sectionnaire, p. 599. II. De la section parisienne à la société rurale: le rôle politique des Parisiens dans les départements, p. 606. - III. - Démocratie directe et égalitarisme primitif : les commissaires civils en campagne, p. 618.

Chapitre VI. - Les armées RÉvolutionNaIRes et la déchristia-

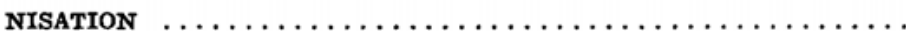

I. - Aspects généraux du problème, p. 636. - II. - Le soldat révolutionnaire et la religion, p. 641. - III. - Déchristianisation spontanée et opérations commandées : l'exemple de l'armée parisienne, p. 658. - IV. - Les armées des départements et la campagne contre les cultes, p. 672. - V. - Appendice: tableau chronologique de la déchristianisation forcée (intervention des armées révolutionnaires), p. 690 .

Chapitre VII. - «Les armées du crime»: vols et pillages,

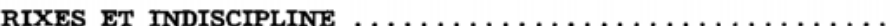

I. - Le procès des armées révolutionnaires devant l'opinion : les origines d'une légende, p. 698 . - II. - Les pillards et les pillages, p. 707. - III. - Les « forces du désordre»: rixes et mutineries, p. 724 .

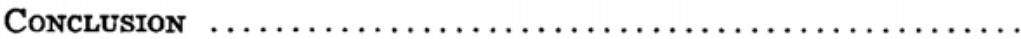




\section{LIVRE TROIS}

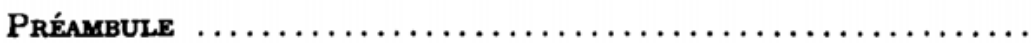

Chapitre PREMTrR. - LA LOI DU 14 Frimaire et LA FIN DRS ARMÉES DÉPARTEMRANTALES ET COMMUNALES (FRIMAIRE-NIVÔSE AN II) .

I. - Le Comité de salut public fixe sa politique (28 brumaire 14 frimaire), p. 748. - I. - La loi du 14 frimaire et la condamnation des institutions populaires, p. 753. - III. - Exécution des mesures de licenciement dans les départements, p. 756. - IV. - Attitude des commissaires civils et des chefs militaires, p. 763. - V. - La première réaction politique dans les départements et l'arrestation des hommes de liaison (21 frimaire - 14 ventôse), p. 767. - VI. $L^{\prime}$ \& amalgame s, les rengagements et le retour à la vie civile, p. 775 .

Chapitre II. - L'armée parisienne en difficulté (NIVôse - ven-

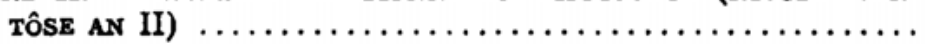

I. - L'armée révolutionnaire et les luttes politiques à Paris (nivôse-pluviôse), p. 781. - II. - Les Lyonnais contre les « étrangers », p. 788. - III. - Les Comités et l'armée parisienne : le Gouvernement révolutionnaire devant le problème de l'autorité, p. 794.

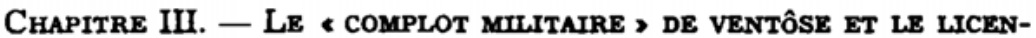
CIEMENT DE L'ARMÉE RÉVOLUTIONNAIRE PARISIENNE (14 VEN-

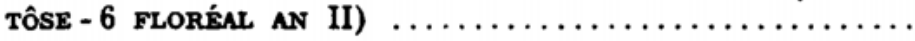

I. - La crise politique de ventôse (14-23 ventôse), p. 809. II. - Le « nouveau Cromwell s : Ronsin dictateur, p. 813. - III. - Les - complices > du général, p. 818. - IV. - L'opinion devant le * complot s: réactions des soldats et des sectionnaires parisiens (24 ventôse - 7 germinal), p. 827. - V. - Les répercussions du * complot , dans les départements (23 ventôse - 22 germinal), p. 836 . - VI. Eloignement des détachements de la région parisienne (23 ventôse 10 germinal), p. 848. - VII. - Le licenciement de l'infanterie et de la cavalerie : le décret du 7 germinal ( 7 germinal - 6 floréal), p. 852 : (1) Le décret du 7 germinal, p. 852; (2) Accueil des révolutionnaires au décret, p. 855; (3) Exécution des mesures de licenciement (9-27 germinal), p. 858; (4) Le sort de l'ancienne artillerie révolutionnaire (germinal an II - prairial an III), p. 867.

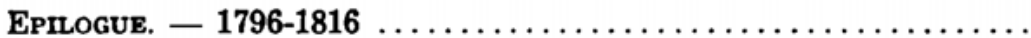

Les cadres survivants, p. 877. - Abonnés aux journaux de Lebois et de Babeuf, p. 878. - Hommes aptes à commander et hommes d̀ surveiller, p. 878. - Le camp de Grenelle, p. 882. - Proscrip- 
tions de l'an VIII, p. 883. - La Machine infernale et les déportations de l'an IX, p. 883 . - Sort des déportés, p. 887. - L'affaire Malet, p. 889. - Le cas Parein, p. 889. - Les ralliements au trône, p. 890. - Le dernier révolutionnaire, p. 891.

NOTES BIBLIOGRAPHIQUES

Archives départementales et communales, p. 897; Archives administratives de la Guerre, p. 924; Archives historiques de la Guerre, p. 927; Archives nationales, p. 930; Archives de la Préfecture de Police, p. 948; Fonds divers, p. 949; Travaux et articles, p. 953.

INDEX DES NOMS DE PERSONNES $\ldots \ldots \ldots \ldots \ldots \ldots \ldots \ldots \ldots \ldots \ldots$

INDEX DES NOMS D'AUTEURS $\ldots \ldots \ldots \ldots \ldots \ldots \ldots \ldots \ldots \ldots \ldots$

INDEX DES NOMS DE LIEUX $\ldots \ldots \ldots \ldots \ldots \ldots \ldots \ldots \ldots \ldots \ldots$

\section{ILLUSTRATIONS}

Les armées démocratiques : élection des officiers et des sousofficiers par les membres de la compagnie (élection de la compagnie de fusiliers de la section du Mont-Blanc, le 29 ven-

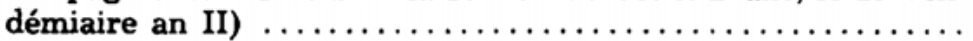

L'humour révolutionnaire: Grammont et le rasoir national (Grammont à Dufresse, le 8 frimaire an II) $\ldots \ldots \ldots \ldots \ldots \ldots \ldots \ldots \ldots$

Les généraux de l'armée révolutionnaire : Parein, Ronsin .......

L'apologie de la Terreur : le soldat parisien et la répression (Lettre de Réaume, datée de Lyon, du 26 frimaire, au comité révolutionnaire de la section de Bonne-Nouvelle) $\ldots \ldots \ldots \ldots \ldots \ldots$

Les candidatures indésirables (lettre adressée au comité révolutionnaire de la section de Bonne-Nouvelle, le 10 octobre 1793, au sujet d'un candidat à l'armée révolutionnaire qui aurait été

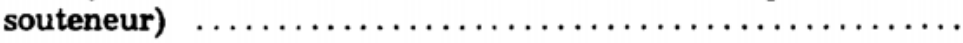

Les fausses armées révolutionnaires (affiche des commissaires civils près l'armée révolutionnaire de la Moselle) $\ldots \ldots \ldots \ldots \ldots$

La campagne contre la ville (affiche des commissaires civils près l'armée révolutionnaire de la Moselle) $\ldots \ldots \ldots \ldots \ldots \ldots \ldots$

L'armée révolutionnaire parisienne et les Sections: Réaume demande des nouvelles de la section de Bonne-Nouvelle (lettre de Réaume au comité révolutionnaire de la section, le 6 ven-

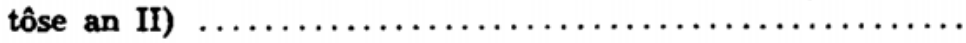

